\title{
EVALUACIÓN DE LA EFICACIA DE LOS CURSOS DE FORMACIÓN SANITARIA DIRIGIDOS A LOS MANIPULADORES DE ALIMENTOS DEL ÁREA SANITARIA DE GANDÍA, VALENCIA $(*)$
}

\author{
Pilar Viedma Gil de Vergara (1), Concha Colomer Revuelta (2) y Lluís Serra Majem (3) \\ (1) Departamento de Higiene de los Alimentos. Centro de Salud Pública de Gandía. Generalitat Valenciana. \\ (2) Unidad de Promoción de la Salud. Escuela Valenciana de Estudios en Salud. Generalitat Valenciana. \\ (3) Área de Medicina Preventiva y Salud Pública. Universidad de Las Palmas de Gran Canaria. \\ $\left(^{*}\right)$ Este trabajo ha sido parcialmente financiado con una beca de la Consellería de Sanitat de la Generalitat Valenciana $\left(\mathbb{N}^{\circ}\right.$ \\ Expediente: 074/1998).
}

\section{RESUMEN}

Fundamento: Las toxiinfecciones por consumo de alimentos contaminados plantean un importante problema de Salud Pública. La formación sanitaria es uno de los mecanismos disponibles para prevenir estas enfermedades. El objetivo de este estudio es evaluar si los manipuladores mejoran sus conocimientos asistiendo a los cursos que se imparten para la obtención del carnet de manipulador y analizar el efecto de las variables sociodemográficas sobre el nivel de conocimientos antes de realizar el curso.

Método: Estudio de evaluación del tipo pre-post, sobre una muestra de 500 manipuladores que acudieron al Centro de Salud Pública de la ciudad de Gandía (Valencia) a realizar el curso de formación, entre octubre de 1997 y febrero de 1998. Se utilizó un cuestionario autoadministrado, antes y después del curso, que medía, entre otras variables, los conocimientos.

Resultados: Se observó diferencia significativa entre los resultados del pre-test y el post-test, excepto para la materia de higiene personal. Los manipuladores con más años de trabajo o con formación sanitaria previa contestaban correctamente con mayor frecuencia.

Conclusiones: Los cursos de formación aumentan el nivel de conocimientos sobre prácticas de manipulación de alimentos. Se debe poner más énfasis, durante la formación, en las materias de conservación, preparación y servicio de alimentos que en las de higiene personal o de instalaciones. No obstante sería interesante estudiar si hay cambios en las actitudes, como una primera aproximación para evaluar la efectividad de la formación y comprobar si hay intención de poner en práctica los conocimientos adquiridos.

Palabras clave: Manipulador de alimentos. Formación. Educación sanitaria. Eficacia. Higiene de los alimentos.

Correspondencia:

Pilar Viedma Gil de Vergara.

Centro de Salud Pública.

C) Duque Carlos de Borja, 19 -

46700 Gandía

Correo electrónico: pilar.viedma@sanidad.m400.gva.es

\section{ABSTRACT}

\section{Assessment of the Effectiveness of Health Training Courses Offered for Food Handlers in a Health Care District}

Background: Food poisoning is a major Health Care issue. Health training is one of the mechanisms available for preventing these illnesses. The purpose of this study is that of ascertaining whether food handlers improve their knowledge regarding food handling practices by attending the courses which are offered for being awarded the handler card and of analyzing the impact of sociodemographic variables on the degree of knowledge prior to taking the course.

Method: Pre-Post type assessment study on a sample of 500 handlers who came to the Public Health Center in the town of Gandia (Valencia) to take a training course in October 1997-February 1998. A self-test questionnaire taken prior to and following the course to gauge knowledge and other variables was used.

Results: A significant difference was found between the pre-test and the post-test, except with regard to the subject of personal hygiene. Those handlers having worked at this occupation for a longer number of years or who had prior health training answered correctly more often.

Conclusións: The training courses heighten the degree of knowledge regarding food-handling practices. In training courses, greater emphasis must be placed on the subjects of food preservation, preparation and serving than on those of personal hygiene or facility cleanliness. Nonetheless, it would be of interest to research whether any changes in attitudes occur as an initial approach to assessing the effectiveness of the training and ascertaining whether any intention exists of putting the knowledge acquired into practice.

Key words: Food handler. Training. Health Training. Effectiveness. Food Hygiene. 


\section{INTRODUCCIÓN}

La Legislación Española ${ }^{1,2}$ define al manipulador de alimentos como aquella persona que por su actividad laboral entra en contacto directo con productos alimentarios o alimenticios destinados al consumo humano.

La formación sanitaria de los manipuladores y las inspecciones a los establecimientos son las dos medidas utilizadas en la prevención de las enfermedades transmitidas por los alimentos. Un grupo de trabajo de la Oficina Regional de la Organización Mundial de la Salud (OMS) para Europa ${ }^{3}$ revisó en 1980 los reconocimientos sanitarios del personal manipulador de alimentos y llegó a la conclusión de que una estrategia alternativa más eficaz que los reconocimientos médicos, sería la educación y formación de quienes trabajan con alimentos y una supervisión y control rigurosos de la higiene de los alimentos, por ejemplo mediante la aplicación de un Sistema de autocontrol, como el llamado Análisis de Riesgos y Control de Puntos Críticos (ARCPC). Así, una alternativa a los exámenes médicos de los manipuladores de alimentos es la formación en prácticas correctas de manipulación de alimentos, entendiendo por tales prácticas aquellas que dieran mayor seguridad de alimentos salubres que un examen clínico. De esta manera, el Reglamento de Manipuladores de Alimentos ${ }^{1}$ incluía la obligación de poseer un carnet que certificara que la persona ha recibido un curso de formación sanitaria sobre prácticas de manipulación de alimentos. El Real Decreto $202 / 2000^{2}$, por el que se establecen nuevas normas relativas a los manipuladores de alimentos, desarrolla la nueva concepción en materia de formación de manipuladores consistente en que las empresas asuman la responsabilidad de desarrollar programas de formación en cuestiones de higiene de los alimentos, este nuevo Real Decreto se reafirma en la poca o escasa utilidad de los exámenes médicos como medio de prevención de enfermedades de transmisión alimentaria y confirma la repercusión favorable de la educación sanitaria en la prevención de este tipo de enfermedades.

Algunos autores ${ }^{4-13}$ se han cuestionado la eficacia de las intervenciones en educación e inspección y han diseñado estudios para evaluarla. Riben ${ }^{14}$ y Mathias ${ }^{15}$ revisaron varios estudios que encontraron en la bibliografía sobre el tema y concluyeron afirmando que la formación de los manipuladores ticne cfecto sobre las puntuaciones de los exámenes y, a corto plazo, sobre las puntuaciones de las inspecciones, pero no pueden definir cual es el proceso educativo más eficaz por las diferencias encontradas en los diferentes estudios revisados en cuanto a diseño y metodología. Finalmente, recomendaban que la formación sanitaria debía continuar.

Este estudio trata de evaluar los cursos de formación sanitaria que se imparten para la obtención del carnet de manipulador de alimentos, no habiendo encontrado ningún estudio similar, realizado en nuestro país, en laš bases de datos IME, MEDLINE, e ICYT. Por otra parte, conocer el nivel de conocimientos de los manipuladores antes de la realización del curso nos facilitaría la labor formativa pudiendo incidir en aquellos aspectos peor conocidos. Asimismo, analizar como influyen las características como edad, sexo, nivel de estudios, formación sanitaria previa, años y lugar de trabajo, sobre los conocimientos antes de la intervención, puede ser útil a la hora de programar y realizar actividades formativas.

Con la incorporación al ordenamiento jurídico español de la Directiva 93/43/CEE, mediante el Real Decreto 2207/1995 ${ }^{16}$, sobre normas de higiene relativas a los productos alimenticios, se empieza a responsabilizar a las empresas de la higiene en sus instalaciones y se obliga a las mismas a realizar actividades de autocontrol. De esta manera se observa un cambio en el concepto de la inspección de industrias de alimentos, de manera que la misión del inspector deja de ser el control y se le empieza a encomendar la vigilancia del autocontrol. Este cambio va a suponer que los Inspectores de Salud Pública tendrán que desempeñar labores de asesoramiento en materia de higiene y seguridad alimentaria.

Los objetivos del presente estudio son: describir la percepción que tienen los manipuladores sobre los inspectores de salud pública; evaluar la eficacia de la formación sanitaria a manipuladores de alimentos, tal y como se está 
desarrollando actualmente; analizar cómo influyen diferentes variables sociodemográficas sobre la adquisición de conocimientos; y cuantificar el número de manipuladores que conoce el sistema ARCPC, sugerido desde todos los sectores como el sistema más adecuado para llevar a cabo el autocontrol en las industrias y establecimientos alimentarios.

\section{MATERIAL Y MÉTODOS}

Se trata de un estudio de evaluación del tipo pre-post. La muestra del estudio comprende 500 manipuladores de alimentos que acudieron al Centro de Salud Pública de Gandía, ciudad costera eminentemente turística situada a unos $65 \mathrm{kms}$ al sur de Valencia, a realizar el curso de formación sanitaria para obtener el carnet de manipulador. El muestreo fue consecutivo durante los meses de octubre del 97 hasta febrero del 98. Los cursos que se realizan actualmente consisten en una charla explicativa y la proyección de un vídeo y tienen una duración aproximada de dos horas. Los monitores que impartieron los cursos utilizaron el mismo material y metodología.

Para la recogida de los datos se han utilizado dos cuestionarios, uno recogía los datos de las variables sociodemográficas, las preguntas sobre la inspección de salud pública y la pregunta sobre el sistema ARCPC y el segundo cuestionario registraba datos sobre conocimientos de prácticas de manipulación de alimentos. Éste último se repetía después de la sesión formativa. Para el diseño del cuestionario sobre conocimientos se revisaron diferentes manuales de prácticas de manipulación de alimentos ${ }^{17-25}$. Las preguntas medían conceptos generales de las siguientes materias: $N B H$ : nociones básicas sobre higiene alimentaria; $C A C$ : compra, almacenamiento y conservación de alimentos; $P S$ : preparación y servicio de alimentos; $H P$ : higiene personal; $H I$ : higiene de instalaciones y utensilios (tabla 1).

Para conocer la percepción que tienen los manipuladores sobre la inspección de salud pública se revisó el estudio realizado por Cunningham ${ }^{26}$ y se escogieron las cinco preguntas que se ajustaban más a la realidad de la inspección local. Estas preguntas, que se describen en la tabla 2, hacían referencia a la forma de trabajar del inspector cuando inspecciona los establecimientos, de manera que cada una de ellas investigaba una faceta del trabajo de un inspector de salud pública: informador; fiscalizador; educador y abierto al dialogo. Se contestaban con una escala de frecuencia que recogía las siguientes posibilidades: frecuentemente, ocasionalmente, nunca y no lo sé. Se preguntó en el cuestionario si conocían el sistema de Autocontrol ARCPC.

Para la evaluación de la eficacia de la formación, la variable dependiente fue la mejora de los conocimientos y las variables independientes fueron: sexo, edad, nivel de estudios, formación sanitaria previa, años y lugar de trabajo.

En la tabla 3 se describen los grupos de cada una de las variables y el porcentaje de la muestra que participa en cada uno de ellos.

A aquellos manipuladores que no sabían leer o escribir se les administraba el cuestionario en forma de entrevista. Se consideró que habían recibido formación sanitaria previa aquellos manipuladores que ya habían acudido a algún otro curso de manipulación de alimentos. Los lugares de trabajo se clasificaron de la siguiente manera: Restauración Colectiva (grupo que incluía bares, cafeterías, restaurantes y comedores institucionales); pastelería; industrias de alimentos (industrias de elaboración de algún tipo de alimentos); otros (aquellos que no estaban recogidos en los grupos anteriores).

Para la confección de la base de datos se utilizó el programa Excel y los cálculos estadísticos se realizaron mediante el programa SPSS para Windows, versión 7.1. Para el análisis de los datos, se aplicó el test de McNemar apareado para detectar diferencias estadísticamente significativas entre las proporciones de respuestas correctas e incorrectas entre el pre-test y el post-test de conocimientos, tomando un nivel.de significación $\mathrm{p}<0,05$. Una vez detectadas las diferencias significativas pre-post, se procedió a realizar un análisis estratificado de dichas diferencias, según las variables sociodemográficas, utilizando la distribución de $\mathrm{Ji}^{2}$. 
Tabla 1

Ítems relativos a conocimientos

\begin{tabular}{|c|l|}
\hline \multicolumn{1}{|c|}{ CONCEPTOS } & \multicolumn{1}{|c|}{ ITEMS } \\
\hline NBH: Nociones Básicas de Higiene Alimentaria & $\begin{array}{l}\text { ¿Qué se entiende por portador sano?: } \\
\text { a) Una persona que está completamente sana; b) Una } \\
\text { persona que alberga microbios en su organismo sin } \\
\text { presentar síntomas de enfermedad; c) Una persona } \\
\text { que tiene predisposición a enfermar. }\end{array}$ \\
\hline $\begin{array}{c}\text { CAC: Compra, Almacenamiento y Conservación } \\
\text { de } \text { Alimentos }\end{array}$ & $\begin{array}{l}\text { A la hora de conservar diferentes alimentos en un } \\
\text { frigorífico, ¿cómo los almacenaría?: } \\
\text { a) Es igual si la nevera está limpia; b) La carne cruda } \\
\text { debajo o lejos de la cocinada; c) Solo me fijaré en la } \\
\text { temperatura. }\end{array}$ \\
\hline PS: Preparación y Servicio de Alimentos & $\begin{array}{l}\text { iCuál de los siguientes alimentos debe conservarse, en frío } \\
\text { si no se va a consumir inmediatamente?: } \\
\text { a) Pollo cocinado; b) Fruta fresca; c) Huevo fresco. }\end{array}$ \\
\hline HP: Higiene Personal & $\begin{array}{l}\text { Los manipuladores de alimentos se lavarán las manos: } \\
\text { a) Con agua caliente y jabón; b) Con agua caliente y } \\
\text { jabón y se las secarán con un trapo; c) Con agua } \\
\text { caliente y jabón líquido y se las secarán con toallas } \\
\text { de un solo uso. }\end{array}$ \\
\hline HI: Higiene de Instalaciones & $\begin{array}{l}\text { ¿Por qué es necesario que los cubos de basura estén } \\
\text { tapados y alejados de la zona donde se manipulan } \\
\text { alimentos?: } \\
\text { a) Porque es más cómodo; b) Porque las basuras son } \\
\text { una fuente de contaminación; c) Porque producen } \\
\text { mal olor. }\end{array}$ \\
\hline
\end{tabular}

Tabla 2

Ítems referentes a la percepción sobre la Inspección de Salud Pública

\begin{tabular}{|c|l|}
\hline $\begin{array}{c}\text { FACETAS DEL TRABAJO DEL INSPECTOR } \\
\text { DE SALUD PÚBLICA }\end{array}$ & \multicolumn{1}{|c|}{ ITEMS } \\
\hline Fiscalizador & $\begin{array}{l}\text { Se fija en si su establecimiento cumple con lo que } \\
\text { dice la ley. }\end{array}$ \\
\hline Informador & $\begin{array}{l}\text { El inspector le indica la reglamentación que se aplica } \\
\text { en su establecimiento. }\end{array}$ \\
\hline Educador & $\begin{array}{l}\text { Le facilita información sobre manipulación correcta } \\
\text { de alimentos. }\end{array}$ \\
\hline Dialogante & $\begin{array}{l}\text { Se toma tiempo en escuchar los problemas de su } \\
\text { establecimiento. }\end{array}$ \\
\hline
\end{tabular}


Tabla 3

Grupos de cada una de las variables estudiadas y porcentaje de la muestra que participa en cada uno de ellos

\begin{tabular}{|cc|cc|}
\hline SEXO & PORCENTAJE & FORMACIÓN S. PREVIA & PORCENTAJE \\
\hline Mujer & $68,2 \%$ & Sí & $73,4 \%$ \\
Varón & $31,8 \%$ & No & $26,6 \%$ \\
& & & PORCENTAJE \\
\hline EDAD $($ AÑOS $)$ & PORCENTAJE & AÑOS DE TRABAJO & $28,6 \%$ \\
\hline$\leq 30$ & $55,8 \%$ & $\leq 1$ & $32,8 \%$ \\
$31-45$ & $30,8 \%$ & $2-5$ & $38,6 \%$ \\
$\geq 46$ & $13,4 \%$ & $\geq 6$ & \\
& & & PORCENTAJE \\
\hline NIVEL ESTUDIOS & PORCENTAJE & LUGAR DE TRABAJO & $44,2 \%$ \\
\hline Ninguno & $2,8 \%$ & Restauración Colectiva & $5,2 \%$ \\
Primarios & $62,2 \%$ & Pastelería & $36 \%$ \\
Secundarios & $10,0 \%$ & Industria alimentación & $14,6 \%$ \\
F.P./Universitarios & $24,9 \%$ & Otros & \\
\hline
\end{tabular}

$\mathrm{N}=500$

\section{RESULTADOS}

\section{Percepción sobre la inspección de salud pública}

El $55,2 \%$ de los manipuladores afirmó haber recibido alguna vez la visita del Inspector de Salud Pública y sólo un 3\% indicó no haber recibido nunca al Inspector. De los 276 manipuladores que afirmaron haber recibido al inspector, más de la mitad contestó que éste frecuentemente se fijaba en si el establecimiento cumplía la Reglamentación (fiscalizador) y el $44,6 \%$ indicó que frecuentemente se le mencionaba qué Ley se le estaba aplicando en la inspección (informador). Solo un 2,5\% afirmó que el Inspector nunca se fijaba en si el establecimiento cumplía la Reglamentación. Sobre si los inspectores facilitaban información sobre cómo manipular alimentos (educador), el 45,3\% afirmó que esto ocurría frecuentemente y solo un tercio indicó que el inspector frecuentemente escuchaba sus problemas (abierto al dialogo). Ver la figura 1.

\section{Análisis de riesgos y puntos críticos} (ARCPC):

De los 500 manipuladores, un $32,4 \%$ no contestó a esta pregunta y de los 338 que contestaron, el 56,5\% conocía el Sistema ARCPC. Conocimientos de los manipuladores sobre
prácticas de manipulación de alimentos:

El 19,6\% de los manipuladores respondió correctamente a todas las preguntas en el pretest, porcentaje que aumenta hasta el $33,8 \%$ en el post-test, con $\mathrm{p}<0,0001$ (test McNemar).

La tabla 4 nos muestra como respondieron a las diferentes preguntas antes y después del curso. Estos resultados son diferentes significativamente para todas las materias, menos para la de higiene personal. La materia con más fallos, antes y después del curso, fue la referida a preparación y servicio de alimentos.

Se realizó un análisis estadístico estratificado según los grupos de las variables sociodemográficas. En la distribución por grupo de edad, el grupo de 31-45 años tiene mejores resultados que el grupo de $\leq 30$ años 
Figura 1

Percepción sobre la Inspección de Salud Pública

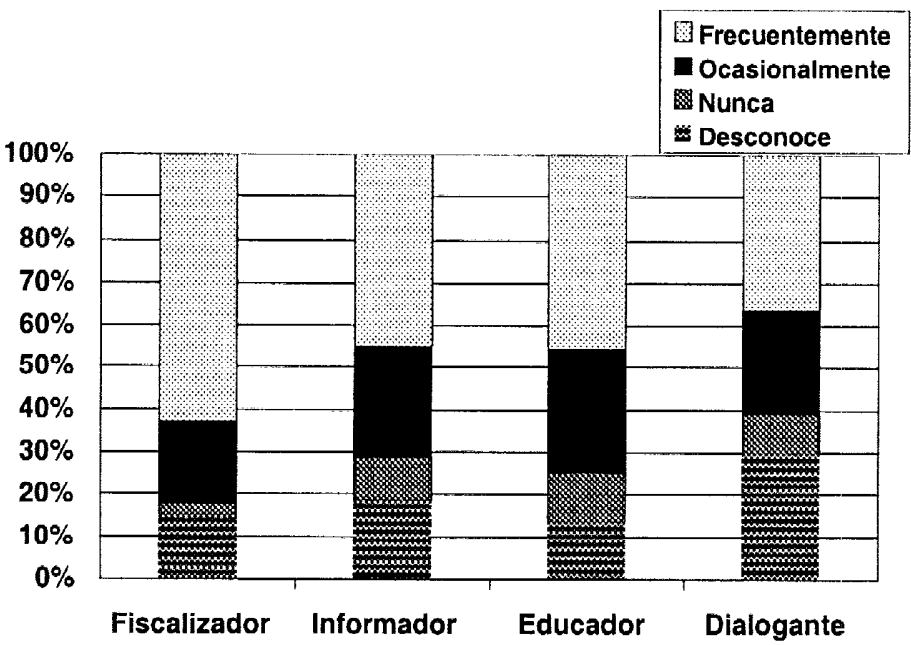

Tabla 4

Como contestaron los Manipuladores a las preguntas sobre conocimientos

\begin{tabular}{|l|c|c|c|}
\hline \multirow{2}{*}{ Áreas temáticas } & \multicolumn{2}{|c|}{$\%$ Correcto } & \multirow{2}{*}{$\begin{array}{c}\text { Diferencia } \\
\text { Significativa }\end{array}$} \\
\cline { 2 - 3 } & Pre-test & Post-test & ( \\
\hline Nociones básicas sobre Higiene Alimentaria (NBH) & $67 \%$ & $80,8 \%$ & $\mathrm{p}<0,0001$ \\
\hline Compra, Almacenamiento y Conservación de Alimentos (CAC) & $65 \%$ & $78,8 \%$ & $\mathrm{p}<0,0001$ \\
\hline Preparación y servicio de alimentos (PS). & $37 \%$ & $50,2 \%$ & $\mathrm{p}<0,0001$ \\
\hline Higiene Personal (HP). & $96 \%$ & $96,4 \%$ & 0,824 \\
\hline Higiene Instalaciones y Utensilios (HI). & $94,4 \%$ & $98,6 \%$ & $\mathrm{p}<0,0001$ \\
\hline Valoración global (5 ítems) & $19,6 \%$ & $33,8 \%$ & $\mathrm{p}<0,0001$ \\
\hline
\end{tabular}

$\mathrm{N}=500$

$(p=0,006)$ y que el grupo de $\geq 46$ años $(\mathrm{p}<0,0001)$; para los tres grupos hay diferencia significativa entre los resultados del pretest y los resultados del post-test $(\mathrm{p}<0,0001 \mathrm{y}$ $\mathrm{p}=0,04)$. En la distribución por sexo, no hay diferencias entre hombres y mujeres antes del curso de formación y en ambos grupos se observa una mejora significativa después de la formación ( $p<0,0001$ para los dos grupos). Según el nivel de estudios, no hay diferencia entre los grupos antes del curso de formación. El curso no hace que mejoren los conocimientos de aquellos manipuladores que han cursado estudios de bachillerato y de aquellos que no tienen estudios, pero para los grupos de estudios primarios y universitarios hay una mejora significativa de conocimientos $(\mathrm{p}<0,0001$ y $\mathrm{p}=0,001)$. Se observa que para los resultados totales del pre-test tienen mejores puntuaciones aquellos que ya habían acu- 
dido a algún curso de formación sanitaria y los dos grupos mejoran significativamente después del curso de formación $(\mathrm{p}<0,0001$ para los dos grupos). En cuanto a los años de trabajo, se observa que los que más años llevan trabajando contestan mejor que los que aún no llevan un año de trabajo $(\mathrm{p}=0,04)$, aunque los tres grupos mejoran significativamente después del curso de formación $(\mathrm{p}<0,0001$ para los tres grupos). No se observan diferencias significativas importantes según el lugar de trabajo para los resultados antes de la formación. Hay diferencias significativas entre pre-test y post-test para todos los grupos $(\mathrm{p}<0,0001$ para restauración colectiva $\mathrm{e}$ industria y $\mathrm{p}=0,001$ para el grupo de otros) menos para los manipuladores del grupo de pastelería $(\mathrm{p}=0,625)$.

\section{DISCUSIÓN}

La evaluación de los cursos de formación, tal y como se ha planteado en este estudio, identifica que éstos aumentan, en general, el nivel de conocimientos respecto a las prácticas de manipulación de alimentos. No se encontró un aumento significativo para la materia de higiene personal. Cuando en higiene alimentaria nos referimos a higiene personal, tratamos cuestiones íntimamente relacionadas con los hábitos higićnicos, como no fumar mientras se trabaja, no comer, no tocar los alimentos con las manos sucias, vigilar la higiene corporal, no toser encima de los alimentos, entre otros. Según los resultados de este estudio, prácticamente todos los manipuladores tienen conocimiento de qué hábitos están considerados poco higiénicos, lo que no quiere decir que no se lleven a cabo. No olvidemos que con este estudio, no pretendemos valorar la efectividad del curso de formación de manipuladores de alimentos como modificador de hábitos, actitudes $y$, por consiguiente, prácticas higiénicas, sino verificar el aumento de conocimientos a corto plazo.

Los años de experiencia y la formación sanitaria previa tienen efecto positivo sobre los conocimientos en la mayoría de las materias, esto nos confirma los resultados obtenidos de mejora de conocimientos tras la formación.
La limitación de la evaluación de la eficacia llevada a cabo con este tipo de estudio, es que el diseño utilizado no es el de mayor poder. Al no existir un grupo control, no se puede concluir que los efectos detectados sean exclusivamente debidos a la sesión formativa. En cualquier caso, lo que sí que se observa después de la formación es un aumento de conocimientos, sin poder demostrar que se deban exclusivamente a la misma.

La elección de los meses de octubre a febrero no es arbitraria. La experiencia nos permite afirmar que la población que acude a los cursos de formación tiene las mismas características durante todo el año, con la excepción de los meses de julio, agosto y septiembre, época del año donde aumenta la demanda de carnets, debido a la apertura de los establecimientos turísticos de verano que emplean a manipuladores temporales.

Los resultados de nuestro estudio son similares a los obtenidos por Nabali ${ }^{9}$ y Cotterchio $^{27}$, que evaluaron la mejora de conocimientos mediante la comparación de las diferencias entre las puntuaciones de un pre-test y un post-test de conocimientos, tras un curso de formación de dos mañanas en el primero y de 15 horas en el segundo. En ambos estudios los cursos de formación se dirigían a los gerentes de los establecimientos de Restauración Colectiva. También son similares a los obtenidos por Kneller ${ }^{11}$; Clingman ${ }^{28}$ y Palmer ${ }^{29}$, que evalúan la efectividad de los cursos de formación comparando las diferencias entre las puntuaciones obtenidas entre una inspección previa al curso y una posterior al mismo, obtienen como resultado que las inspecciones tienen puntuaciones más favorables después de la formación.

En cuanto a la percepción que tienen de la inspección de Salud Pública los manipuladores de alimentos, estas preguntas hacían referencia a cada una de las facetas del trabajo de un inspector: informador; fiscalizador; educador $y$ abierto al dialogo. Según nuestro estudio, los manipuladores perciben, con mayor frecuencia, a los inspectores como alguien que va a comprobar si sus instalaciones cumplen con la reglamentación, más que como alguien que les va a facilitar información o ayuda técnica. 
Debemos reflexionar sobre esto, porque una buena comunicación entre inspector y manipulador se ve necesaria para poner en práctica los conocimientos adquiridos con la formación y para que la administración vigile y controle que efectivamente se llevan a cabo unas buenas prácticas de manipulación de alimentos, tal y como marca la legislación ${ }^{16,30}$.

Hay que destacar el hecho de que las dos materias con porcentajes más bajos de respuestas correctas, antes de la formación y después de la misma, fueron las referidas a preparación y servicio y a compra, almacenamiento y conservación de alimentos, aspectos que frecuentemente se relacionan con los brotes de toxiinfección alimentaria. Según Bryan ${ }^{2}$ los factores contribuyentes de las toxiinfecciones son: refrigeración inadecuada, conservación en caliente a temperatura incorrecta, cocinado incorrecto y sucesivos recalentamientos de los alimentos. A pesar de que se observan diferencias significativas entre los resultados del pre y el post-test, es en estas materias, donde se tenía que haber observado mayor porcentaje de respuestas correctas después de la intervención formativa y observamos que la materia de preparación y servicio no aumenta más que hasta un $50,2 \%$ de respuestas correctas. Tenemos que reflexionar sobre los contenidos de los programas formativos que se están llevando a cabo actualmente y afirmar que cn los cursos de formación sanitaria se debería dar más importancia a esas cuestiones que a las referidas a higiene personal o de instalaciones, materias mejor conocidas por los manipuladores antes de recibir la formación. Puesto que una sesión formativa de dos horas se ha manifestado suficiente para aumentar significativamente los conocimientos, habría que aprovechar este tipo de formación para obtener mejores resultados. Los conceptos que deberían impartirse en los cursos de formación deberían hacer referencia a posibles causas de contaminación de alimentos, prevención y eliminación de la misma, y en relación con esto la influencia de la refrigeración, congelación y cocinado. En la materia de higiene personal lo interesante sería introducir el concepto de la relación entre unos hábitos poco higiénicos y la posibilidad de que se contaminen los alimentos que pudieran estar manipulando, resaltar el hecho de que un manipulador de alimentos es una posible fuente de contaminación de los alimentos que manipula.

Por otro lado tener buenos conocimientos sobre manipulación de alimentos no garantiza una manipulación correcta, por eso se hace necesario introducir, en futuros estudios, mecanismos de evaluación de las actitudes, como una primera aproximación a la evaluación de la efectividad de la formación sanitaria.

Es importante también que en futuros estudios se valide la pregunta sobre el ARCPC, dado el elevado porcentaje de manipuladores que dicen conocerlo.

\section{AGRADECIMIENTOS}

Queremos agradecer a $D^{a}$ Julia Martínez la colaboración prestada a la hora de impartir los cursos y al Departamento de Epidemiología del Centro, en especial a D. Amancio Peñuelas, la ayuda prestada en el análisis de los datos.

\section{BIBLIOGRAFÍA}

1. Boletín Oficial del Estado. Real Decreto de la Presidencia del Gobierno 2505/1983, de 4 de agosto, por el que se aprueba el Reglamento de manipuladores de alimentos. BOE núm. 225, 20/09/1983.

2. Boletín Oficial del Estado. Real Decreto $202 / 2000$, de 11 de febrero, por el que se establecen las normas relativas a los manipuladores de alimentos. BOE núm. 48, 25/02/2000.

3. Bryan FL. Hazard Analysis Critical Control Point Aproach: Epidemiologic Rationale and Aplication. J Environ Health 1981; 44: 7-14.

4. Riben PD, Mathias RG, Wiens M, Cocksedge W, Hazelwood A, Kirshner B, Pelton J. Routine restaurant Inspections and Education of Food Handlers: Recommendations Based on Critical Appraisal of the Literature and Survey of canadian Jurisdictions on Restaurant Inspections and Education of Food Handlers. Can J Public Health 1994; Suppl.1: S67-S70.

5. Mathias RG, Sizto R, Hazelwood A, Cockscdgc W. The Effects of Inspection Frequency and 
Food Handler Education on Restaurant Inspection Violations. Can J Public Health 1995; $86 \mathrm{~N}^{\circ} 1$ : 46-50.

6. Irwin K, Ballard J, Grendom J, Kobyashi J. Results of Routine Restauran Inspections can predict outbreaks of Foodborne Illness: The Seattle-King County Experience. Am J Public Health 1989; 79, 586-90.

7. Corber S, Barton P, Nair RC, Dulberg C. Evaluation of the Effect of Frequency of Inspection on the Sanitary Conditions of Eating Establishments. Can J Public Health 1984; 75: 434-38.

8. Bader M, Blonder E, Henriksen J, Strong W. A Study of Food Service Establishment Sanitation Inspection Frequency. Am J Public Health 1978; 68: 408-10.

9. Nabali H, Bryan FL, Ibrahim J, Atrash H. Evaluation of Training Food Service Managers in Bahrain. J Environ Health 1986; 48: 315-18.

10. Stevenson RK. A Food Service Establishment Evaluation Program Procedure for the 1980's and 1990's. J Environ Health 1987; 50: 25-8.

11. Kneller P, Bierma T. Food Service Certification. J Environ Health 1990; 52: 292-94.

12. Penman $\mathrm{AD}$, Webb RM, Woernle $\mathrm{CH}$, Currier MM. Failure of Routine Restaurant Inspections: Restaurants-Related Foodborne Outbreaks in Alabama, 1992, and Missisipi, 1993. Environmental J 1996; April: 23-25.

13. Travis HR. Training for Seasonal Foodservice Operations. J Environ Health 1986; 48: 265-67.

14. Riben PD, Mathias RG, Campbell E, Wiens M. The Evaluation of the Effectiveness of Routine Restaurant Inspections and Education of Food Handlers: Critical Appraisal of the Literature. Can J Public Health 1994; 85 Suppl.1: S56-S60.

15. Mathias RG, Riben PD, Campbell E, et al. The Evaluation of the Effectiveness of Routine Restaurant Inspections and Education of Food Handlers: Restaurant Inspection Survey. Can J Public Health 1994; 85 Suppl.1: S61-S66.

16. Boletín Oficial del Estado. Real Decreto $2207 / 95$, de 28 de diciembre, por el que se establecen las normas de higiene de productos alimenticios. BOE núm. 50, 27/02/96.

17. Gobierno Vasco. Departamento de Sanidad y Consumo. Dirección de Salud Pública. Higiene Alimentaria: Una Nueva Estrategia de Formación de Manipuladores. Vitoria-Gasteiz: Departamento de Sanidad y Consumo; 1990.
18. Ministerio de Sanidad y Consumo. Subdirección General de Higiene de los Alimentos. Manual de Higiene Alimentaria. Madrid: Ministerio de Sanidad y Consumo;1991.

19. Generalidad Valenciana y Ministerio de Sanidad y Consumo. Consejería de Sanidad, Trabajo y Seguridad Social. Manual para Manipuladores de Alimentos. Madrid: Generalidad Valenciana y Ministerio de Sanidad y Consumo; 1983.

20. Generalidad Valenciana. Consejería de Sanidad y Consumo. Manual para Manipuladores de Alimentos. Valencia: Consejería de Sanidad y Consumo; 1990.

21. Gobierno de Canarias. Consejería de Sanidad y Consumo. Manual para Manipuladores de Alimentos. Santa Cruz de Tenerife: Consejería de Sanidad y Consumo; 1993.

22. Gobierno de la Comunidad Autónoma de Madrid. Consejería de Sanidad y bienestar Social. Manuales para Manipuladores de Alimentos. Madrid: Consejería de Sanidad y bienestar; 1996.

23. Diputación General de Aragón. Departamento de Sanidad, Bienestar Social y Trabajo. Manual para Manipuladores de Alimentos. Zaragoza: Diputación General de Aragón; 1991.

24. Hazelwood D, McLean A D. Curso de higiene para manipuladores de alimentos. Zaragoza: Editorial Acribia, SA; 1991.

25. Jacob M. Manipulación correcta de los alimentos: Guía para gerentes de establecimientos de alimentación. Ginebra: Organización Mundial de la Salud;1990.

26. Cunningham CJ. A Survey of the Attitudes and Perceptions of Food Service Operators in the Hamilton-Wentworth Region. Can J Public Health 1993; 84, N2:107-11.

27. Cotterchio M, Gunn J, Coffill T, Tormey P, barry'A. Effect of a Manager Trainig Program on Sanitary Conditions in Restaurants. Public Health Reports 1998; 113: 353-58.

28. Clingman CD. Ohio Evaluates Effects Of Food Certification Training. J Environ Health 1976; 38: 235-36.

29. Palmer BJ, Hatlen JB, Jackson BB. The Implementation And Evaluation Of Management Training In Fast Food Restaurant Chains. J Environ Health 1975; 37: 364-68.

30. Boletín Oficial del Estado. Real Decreto 50/93, de 15 de enero, sobre Control Oficial de los productos alimenticios. BOE núm. 36, 11/02/93. 